\title{
LA BIOJURÍDICA O EL BIODERECHO COMO MEDIADOR DE LOS NUEVOS DILEMAS BIOMÉDICOS
}

\author{
Biojuridics or biolaw as a mediator of the new biomedical dilemas
}

\author{
Juan Carlos Araujo-Cuauro*
}

\section{RESUMEN}

El avance de la bioética como ciencia ha excedido el escenario ético para sumergirse, plenamente, en otros entornos del saber, esencialmente en la esfera de lo legal, como lo es, lo biojurídico y hacia un nuevo protagonismo de una ciencia emergente como lo es el bioderecho. Existe dilemas o premisas bioéticas de donde generan las grandes interrogantes jurídicas, en torno a las ciencias del derecho, las cuales se deben ajustar a la realidad social generada por los grandes avances biotecnológicos, con el fin de regular el comportamiento generado por la conducta humana. El objetivo de la investigación es hacer un análisis sobre el papel que desempeña la bioética, en el desarrollo de la biojurídica o bioderecho como mediador de los nuevos dilemas biomédicos. La metodología empleada es una investigación documental bibliográfica donde se enfocan las posturas de algunos autores como Aparisi (2007) y Schaefer (2017), entre otros. Se realizó una revisión documental desde una postura crítica de las fuentes literarias seleccionadas y empleadas para desarrollo de la investigación, esto con la finalidad de identificar las diversas concepciones que han tenido los avances biotecnológicos sobre la conducta humana y su necesidad de ser regulado por el derecho. Se concluye que la relación entre la bioética y el derecho ha sido muy provechosa, al momento de dar repuesta aquella temática del debate de los grandes dilemas bioéticos, lo que da como resultado, el surgimiento de una novedosa rama jurídica como lo es la biojurídica o bioderecho.

Palabras claves: Bioética, biojurídica, bioderecho, problemas, médicos legales.

RECIBIDO: Enero 2019

ACEPTADO: Junio 2019

\footnotetext{
* Médico y Abogado. Profesor de Medicina Legal. Facultad de Ciencias Jurídicas y Políticas. Escuela de Derecho Universidad del Zulia (LUZ). Maracaibo, Venezuela. Correo electrónico: jcaraujoc_65@ hotmail.com; jcaraujoc95@gmail.com Código ORCID 0000-0002-6559-5370. Google Scholar https://scholar.google.es/citations?user=_Jvaga8AAAAJ\&hl=es

591
} 


\section{ABSTRACT}

The advance of bioethics as a science has exceeded the ethical scenario to fully immerse oneself in other environments of knowledge, essentially in the sphere of the legal, as it is, the bio-legal and towards a new protagonist of an emerging science such as the biolaw. There are dilemmas or bioethical premises from which generate the great juridical questions, around the legal sciences, which must be adjusted to the social reality generated by the great biotechnological advances, in order to regulate the behavior generated by human behavior. The objective of the research is to analyze the role of bioethics in the development of biolaw or biolaw as a mediator of new biomedical dilemmas. The methodology used is a bibliographic documentary research which focuses on the positions of some authors such as Aparisi (2007) and Schaefer (2017), among others. A documentary review was made from a critical stance of the literary sources selected and used for the development of the research, this with the purpose of identifying the different conceptions that biotechnological advances have had on human behavior and its need to be regulated by law. It is concluded that the relationship between bioethics and law has been very useful, at the time of responding to the theme of the debate on the great bioethical dilemmas, which results in the emergence of a novel legal branch such as bio-legal or biolaw.

Keyword: Bioethics, biolaw, biolaw, problems, legal doctor.

\section{Introducción}

Después de cuatro décadas de su nacimiento u origen la bioética, como ciencia se consagra hoy en día en todo el mundo moderno, en un sitio privilegiado en la tendencia actual de la sociedad, debido a los conocimientos y las contribuciones intelectuales, como son las discusiones libres que ésta disciplina nos encauza, lo cual ve permeado por la justa inquietud sobre el progreso de la ciencia médica en el campo de las tecnologías y la ética en sus probables empleos, este desasosiego ha rebasado el entorno de la sociedad, la de los investigadores y trabajadores de la salud, llegando a otras disciplinas afines, como a la sociología, la filosofía, la política, la ciencia jurídica con referencia a lo legislativo (Gracia, 1989).

La ciencia jurídica basándose en ese orden jurídico viene a formar parte de este armazón que compone a la vida social, éste da forma y estructura a la sociedad y cimienta sobre valores internos, como resultado el derecho como ciencia dinámica debe de considerar la producción de normas para orientar y resolver las diferentes controversias entre los diversos valores e intereses de los individuos de una sociedad. 
La bioética y el bioderecho son dos ciencias que han tenido un gran acercamiento en los últimos tiempos, ambas disciplinas han impactado en los recintos legislativos, en la búsqueda de que los legisladores, los responsables de crear las leyes, las cuales intervienen en la vida cotidiana de todos y en el futuro de la sociedad, lo hagan con la perspectiva de crear leyes justas, bajo el estricto contexto de dar a cada ciudadano lo que le es suyo, lo que le corresponde (Aparisi, 2007).

La bioética en este sentido, aporta a la ciencia jurídica y al derecho parlamentario, el análisis de la realidad concreta, centrado en un análisis ético sobre la vida basado en el estudio de las ciencias médicas, sino en todas aquellas disciplinas y ciencias que interactúan en la vida humana. Mientras que a su vez el derecho como ciencia social, actúa en la razón de la legitimización de las leyes.

El desarrollo bioético ha tenido una gran influencia e incidencia en el ámbito del derecho. Indudablemente, la determinación de lo admisible, en relación a los numerosos modos de influir en el fenómeno de la vida, no es un asunto que perjudica sólo a la ética, sino también, que cada día con mayor magnitud e intensidad, al derecho.

En existencia, podría llegar a enunciar que no existe otro ámbito en la sociedad, como el jurídico, en el que las controversias que, inicialmente, se plantean como bioética o biojurídica, presenten tanta derivación e impacto en las consecuencias médico legales, como las cuestiones bioéticas de mayor calado o trascendencia social, transforman, en muchos casos, en problemas jurídicos que vienen a ser abordados por el bioderecho.

El objetivo de la investigación es hacer un análisis sobre el papel que desempeña la bioética, en el desarrollo de la biojurídica y por ende el nacimiento del bioderecho en el abordaje de los dilemas bioéticos producto del avance de la biotecnología los cuales generan grandes interrogantes jurídicas.

Así como la metodología empleada para lograr el objetivo propuesto, se realizó una revisión documental desde una postura crítica de las diversas fuentes literarias 


\section{La biojurídica o el bioderecho como mediador de los nuevos dilemas biomédicos}

seleccionadas y empleadas para desarrollo de la investigación, esto con la finalidad de identificar las implicaciones ético morales que han tenido los avances biotecnológicos sobre la conducta humana y su necesidad de ser regulado por el derecho a través del surgimiento de unas de su rama como lo es el bioderecho para dar respuesta a las grandes interrogantes jurídicas que surgen con la bioética de los dilemas biomédicos modernos; lo que se inician la investigación biomédica, algunos temas concretos como los problemas del inicio de la vida y del final de la vida .

Entonces para entender la relación entre la bioética y el bioderecho, ambas disciplinas no están solo para la aprobación de las cuestiones que tienen relación con el comportamiento humano ejecutado o por ejecutar, la promoción de nuevas normas en beneficio de la humanidad, por lo que la bioética y la biojurídica o bioderecho tienen que tener una coherencia entre sí, que permitan su avance en su aplicación simultáneamente para garantizar los derechos humano donde estén involucrado el derecho a la vida, el libre desenvolvimiento de la personalidad y los derechos después de la muerte (Cornejo, 2015).

Se hizo un estudio documental recurriendo a las fuentes primarias en la recopilación de la información a través de las fuentes bibliográficas de los procesos de indagación, reflexión, construcción y re-significación del conocimiento sobre los dilemas bioéticos en relación a la investigación biomédica y su relación con el bioderecho.

\section{Los orígenes de la bioética: su nacimiento como ciencia humana}

A raíz de los grandes avances tecnológicos en el área de las ciencias de la salud, en los años setenta y ochenta surgió una nueva disciplina en la que convergen tres ciencias principalmente: La Filosofía, la Medicina y el Derecho. A esta disciplina denominada Bioética. El término bioética define como el estudio sistemático de la conducta humana en el campo de la ciencia de la vida y la salud, analizada a la luz de los valores y principios morales (Maldonado, 2007). 
Sin embargo, esta sigue siendo hoy por hoy, a pesar de sus cuatro décadas de historia, un tema desconocido, inclusive en la esfera de lo académico; asimismo el concepto de bioderecho, por ende, sufre esta misma suerte de condición de desconocimiento. Pero el hecho cierto es que hay problemas y circunstancias que son objeto de reflexión por parte de la bioética.

La bioética como ciencia humana ha logrado una autonomía académica erigiéndose actualmente desde dos vertientes la primera como una disciplina científica, y la segunda como respuesta de los movimientos sociopolíticos y culturales a los avances procedente de las nuevas tecnologías surgidas en la segunda mitad del siglo XX, derivado del fenómeno aplicado a la ética médica para afrontar los problemas médico legales relacionados con el principio y el final de la vida de las personas y las exigencias éticas derivadas de su protección. Su repercusión sobre el derecho plantea exigencias específicas relacionadas con los procedimientos de células madre, el embrión, con la maternidad subrogada, la muerte, la eutanasia, entre otros (Parra, 2006).

En este orden, la bioética no debe ser considerada como una disciplina aislada de otras áreas del conocimiento como en el área del derecho, ya que es una palabra que está realmente comprendida o vinculada a otros vocablos como lo son, la moral, la ética, la deontología, la ética biomédica y hoy en día a la biojuridica o bioderecho (Celi Frugoni, 2015).

Igualmente, la bioética es, una disciplina específicamente ética, en este sentido, se ha catalogado a la bioética formalmente como una rama o subdisciplina del saber ético, del que recibe el estatuto epistemológico básico y con el que mantiene una relación de dependencia justificadora y orientadora.

Desde su origen la bioética ha evidenciado un privilegió en el paradigma principialista, el cual viene amparar de forma general el incremento de reglas de acción sobre la vida, que favorece una tendencia mecanicista de la bioética, lo que conduce a una exorbitante objetivación de las eventualidades de acción, en donde está incluido el 


\section{La biojurídica o el bioderecho como mediador de los nuevos dilemas biomédicos}

ser humano en dicha objetivación (Gracia, 1989).

Bajo esta perspectiva, la bioética limita a un plano de normatividad condicionada a la práctica y muy descuidado en el plano de lo legal con respecto a la dignidad humana; en circunstancias que ella es también, y primariamente, reflexión en cuanto a la exigencia fundamental del comportamiento de las personas.

No basta con establecer, cómo se debe actuar, o sea, enunciar normas jurídicas y de acción. También como ciencia le interesa exhibir, mostrar por qué actuar de esa forma, es decir, establecer con una adecuada fundamentación más vigorosa basada en los principios de acción ética (Aparisi, 2007).

Los seres humanos como individuos son sujetos éticos, de derechos y deberes, son individuos respetado (éticamente) y tutelado (jurídicamente), y es lícito todo lo que no daña a la persona e ilícito todo lo que la suprime o daña.

Esto significa que las personas no pertenecen a una categoría de bienes útiles o instrumentales, no son un producto, una persona tiene más valor que una cosa siempre y cuando con dicha acción posterga a la primera frente a la segunda, por lo que tal actuar es incorrecto.

La dignidad de las personas es fundamentada en la exigencia del máximo respeto y de una efectiva tutela, en el campo de la bioética, desde el momento de la concepción hasta el momento de la muerte natural, y siempre que muestre necesitada de ayuda.

Es por ello que a partir de este hecho cabe decir que la persona es un continuum, vocablo que nos permite entender y explicar que ella es la misma desde el momento en que comienza su existencia hasta el momento de su muerte; Es la misma que ha pasado por una fase embrionaria, fetal, de recién nacido; Es la misma que ha ido a la sala maternal, al colegio, a la universidad; que ha trabajado como profesional, se ha casado, ha envejecido, ha enfermado y ha caído en estado de coma o que ha perdido el sentido de la realidad (Martínez, 2013).

En la actualidad, el desarrollo de la bioética ha rebasado el marco ético para introducirse, plenamente, en otros ámbitos del saber, especialmente en el jurídico; 
debido a que, en la realidad social moderna no existe otro campo como el derecho, en el que las controversias que, inicialmente, se plantean como bioéticas, presenten tanta derivación e impacto.

En el contexto del derecho médico venezolano muchas veces se encuentra en una encrucijada debido a la problemática de la no existencia de fundamentos jurídicobioéticos. Quizá sea porque aún no llega el momento o que simplemente no se considere importante para empezar a realizarlo, pues en Venezuela existe el basamento legal para la protección de la vida, como lo contempla el texto constitucional en su artículo $43^{\circ}$ que señala que: "El derecho a la vida es inviolable... Omissis".

$\mathrm{Al}$ apreciar el desarrollo bioético ha tenido una gran incidencia en el campo de la ciencia del derecho, debido a las grandes situaciones controversiales que este debe enfrentar. Por lo que puede llegar a reconocer que no existe otro campo, como el jurídico, en el que las controversias que, inicialmente, plantean como bioéticas, presenten tanta derivación e impacto en lo jurídico-legal (Aguiar-Guevara, 2008).

Son precisamente aquellas cuestiones bioéticas de mayor trascendencia social, las que transforman en muchas ocasiones en problemas jurídicos. Es evidente que la acción humana en este caso tecnificada, puede producir daños, incluso irreversibles, a las personas. En consecuencia, la respuesta a esta realidad no debe tener, sólo carácter moral, ni reducirse, por tanto, a una cuestión privada, sino que debe tener una respuesta jurídico-legal (Gutiérrez Samperio y Vila-Coro 2007).

La controversia siempre estará centrada, precisamente, en la determinación de los límites de la frontera entre la bioética y el bioderecho y, en definitiva, entre la moral y el derecho. No obstante, como ya se ha manifestado con anterioridad, al establecer la frontera entre bioética y bioderecho habría de tener en cuenta ciertas peculiaridades. En primer lugar, es evidente que, desde un punto de vista histórico, la reflexión bioética es anterior al bioderecho ya que dicha reflexión bioética aporta datos y reflexiones metodológicas formidablemente útiles al bioderecho. 
Por ello, como lo ha señalado Gracia, "el bioderecho sin bioética es ciego, y la bioética sin el bioderecho resulta vacía. Sin la bioética, el bioderecho correrá siempre el riesgo de caer "bajo mínimos" (Gracia, 1989, p. 576).

Entonces la bioética fue la excepción, cuando los conflictos médico legales entre médicos y pacientes, o sus familiares, originado por la aplicación de determinados procedimientos diagnóstico y/o terapéutico, o práctica clínica, no lograban ser resueltos en el ámbito del consultorio, trasladaban a los tribunales, dando inicio a una vasta colección de decisiones jurisprudenciales, como de la doctrina dedicada a la solución de los problemas bioéticos a generar.

Sin embargo, las nuevas tecnologías a medida de su avance junto a la experimentación, surgían otros problemas los cuales directamente afectan los derechos de las personas, entonces la bioética llamada jurídica constituye una nueva forma de afrontar la búsqueda de solución a los conflictos planteados en esta era moderna, es el conjunto de normas jurídicas de interés público se encuentran íntimamente ligadas a la protección de la vida desde su inicio hasta su final respetando la dignidad humana (Flores, 2004).

\section{Una mirada a la relación entre la ética, la bioética y el derecho}

Desde sus propios comienzos, la ética, como la bioética se ha caracterizado por sus fuertes y estrechos vínculos con el derecho, por ello, al entender y comprender de una forma, aunque sucinta, las similitudes y las disconformidades entre ambas ciencias, lo cual es fundamental para entender su fuerte interrelación, basada en importantes influencias recíprocas, son tan necesaria para dar respuesta a la nuevas cuestiones jurídicas, se presentan debido al avance acelerado del desarrollo biotecnológico genera una serie de dilemas bioéticos, originando consecuencias biojurídicas, han permitido el desarrollo del "bioderecho o biolaw" (Schaefer, 2017).

Si bien ética y derecho son disciplinas diferentes, se encuentran internamente relacionadas, con respecto a dicha relación sus enunciados se orientan a respetar la autonomía de cada uno de los dos ámbitos, pero de tal manera que acceda su influencia 
recíproca: de la ética como petición crítica del derecho y del derecho como declaración afirmativa y práctica de la ética".

El planteamiento que hace referencia a la correlativa influencia de la ética, a la bioética, y del derecho al bioderecho. El neologismo bioderecho, más aún, por momentos habla indistintamente de derecho o de bioderecho en su relación o vínculo con la bioética (Hooft, 2002).

El bioderecho constituye una nueva forma de solución a los conflictos, es el conjunto de normas jurídicas de interés público ligado íntimamente a la protección de la vida desde el nacimiento hasta la muerte, pero con un respeto a la dignidad humana. Este término confundido, por algunos autores en donde afirman que el derecho se relaciona con el concepto de biología; quizá como ciencia, al relacionarlo, mas no en el ámbito gramatical, la Bioética es la parte troncal en la concepción natural del bioderecho (Celi Frugoni, 2015).

El bioderecho viene a solucionar los conflictos desde el planteamiento ético, con el soporte o aval de la ciencia y bajo el marco de un derecho cercano a la sociedad cuyo referente último radica en el imperativo sustentado por los Derechos Humanos (Casado, 2002).

La ciencia ha mostrado un experimentado e impresionante desarrollo desde el acontecer de la biotecnología, de una manera muy especial desde los finales del siglo $\mathrm{XX}$, a los inicios del Siglo XXI cuyo no inimaginables pocas décadas atrás, estas estuvieron marcadas por cuestiones enlazadas con la libertad científica, un impresionante y un extraordinario progreso biotecnológico científico, la protección de la vida humana, la búsqueda del equilibrio ambiental, la socialización y la universalización de la salud, y el reconocimiento de la autonomía del paciente, ha posibilitado en las cuatro últimas décadas, llevar a cabo producto de las exigencias de la sociedad juntos a sus individuos una revisión de los establecimientos clásicos de Derecho (Flores, 2004 y Schaefer, 2017). 
El objeto principal de la investigación científica ha sido el ser humano, la vida humana en todas sus facetas temporales, han alcanzado tales niveles de intervención en la entidad constitutiva de la persona y en la tecnificación del entorno humano, la repercusión que ello ha tenido a nivel social probablemente no es comparable a ninguna otra de las conquistas científicas (Moran, 2006).

Es por ello el bioderecho ha querido erigirse como la esfera del derecho regulador de las nuevas tecnologías en la biomedicina surgidas con la sociedad posmoderna.

Su interés es eminentemente normativo y, por consiguiente, negativo, puesto se concentra en el estudio de los riesgos, peligros y amenazas que las nuevas biotecnologías sobre en el campo de la medicina aplicadas a los seres y sistemas vivos implican o acarrean situaciones problemáticas (Maldonado, 2007).

Bajo este concepto podemos encontrar todo aquello que ponga en riesgo la vida, la libertad, la dignidad y los demás principios sean definidos como los rectores de la sociedad moderna los cuales se vuelven objeto de regulación normativa y jurídica. Es necesario no olvidar y tener siempre en cuenta uno de los fines del derecho, es marcar los causes jurídicos adecuados en las relaciones interpersonales de los individuos, transcurran en un marco de orden para evitar los conflictos de intereses (Vila, 2005).

Por lo tanto, en este nuevo rol el derecho debe abordar los temas bioéticos y los nuevos conceptos jurídicos, como el de persona, están en plena reelaboración, con el ánimo de trazar los lineamientos para que exista compatibilidad entre los avances biotecnológicos y de los valores ético morales, donde deban asegurar y respetar el reconocimiento por la humanidad (Parra, 2006).

En este punto es necesario y saludable enfatizar que la mayoría de todos estos problemas pueden ser engendrados, donde existe un componente muy importante de índole ético-moral los cuales no son abstraído de la realidad.

No obstante, el valor de la vida, la dignidad de la persona, son realidades expuesta o planteadas desde un campo moral y ético, debe considerarse con todo el respeto, se convierten en la voz de una sociedad donde todavía mantienen su moral 
como guía de comportamiento, y aunque la bioética no puede convertirse en una moral, está se va a nutrir y fortalecer de la misma.

El bioderecho o la biojurídica es la respuesta desde el mundo del derecho al surgimiento de la bioética. El bioderecho sistematiza y da coherencia jurídico-ética a las nuevas realidades emergentes modernas sobre la vida y la sociedad como figuras jurídicas poco observadas y con abismos legales en el marco jurídico del derecho médico venezolano (Aguiar-Guevara, 2008).

Es por ello el bioderecho es un área de la justicia compuesta por los diversos casos y soluciones producidas particularmente en el desarrollo de la vida humana. Para entender y por si comprender el campo de acción del bioderecho es necesario tener constantemente presentes las vicisitudes dadas entre la vida humana, los fenómenos de crisis y de "revolución", quizás los más grandes de todo el desarrollo humano, generado por el sorprendente impacto técno-cientifico actual.

El bioderecho es una nueva rama científica interdisciplinaria, dotado con objetivos y principios y con finalidades propias dedicada abordar de manera inédita y novedosa el intrincado y apasionante campo relacionado con la vida, con la reproducción humana y la muerte determinando sus alcances y sobre todo sus límites (Flores, 2004).

Las ideas y reflexiones expresadas conllevan a plantear cuales son los retos a enfrentar por el bioderecho. Lo primero que debe enfrentar el bioderecho es la necesidad para acometer un diálogo interdisciplinario con otras áreas del saber. Este respectivo diálogo no debe tener como propósito doblegar un saber en favor de otro; su propósito debe ser el de enriquecer la visión de los múltiples fenómenos y problemas sobre los cuales nos movemos en la actualidad cotidiana.

Asimismo, el bioderecho hoy debe dialogar con ciencias como la medicina, la ingeniería, la robótica, la nanotecnología, entre otras., muchos de los problemas surgen como objeto de su interés en dichas ciencias. 
El bioderecho, en muchas situaciones, surge como respuesta a los avances del progreso científico y tecnológico vinculados con la problemática de los seres vivientes, y representa una simbiosis entre la vida y el comportamiento humano en su entorno natural, relacionado con todo aquello relativo a la vida y la dignidad.

Son muchos los campos del accionar del bioderecho, debido a los grandes dilemas bioéticos biomédicos, originan grandes interrogantes jurídicas, producto de los avances biotecnológicos científicos, dan nacimiento a esta ciencia del derecho con el fin de regular el comportamiento generado por la conducta humana, dado en la mayoría de los casos, no existen parámetros para juzgar los asuntos biomédicos modernos referentes al principio o inicio de la vida humana como; la eugenesia, fertilización asistida médicamente, genoma humano, embriones, ensayos clínicos con células madres, fecundación postmorten, experimentación en humanos, parto humanizado, maternidad subrogada, dignidad, optimización de recursos en medicina intensiva, utilización o no del soporte vital artificial, muerte digna, autonomía, consentimiento informado, acceso a las nuevas tecnologías en procedimientos, en terapias, en medicamentos, negación a recibir transfusión sanguínea por los testigos de Jehová, la adopción de embriones, entre tantas otras. Es decir, la evolución de la biomedicina: nuevas fronteras, nuevos dilemas.

Asimismo, el bioderecho en el final de la vida humana como el aborto, la píldora del día después, la eutanasia y sus diferentes figuras, criogenización, la donación y trasplantes de órganos, tejidos y células, el paciente terminal. $\mathrm{O}$ de otras aplicaciones científico-tecnológicas en asuntos tan inquietantes como la ingeniería genética humana y animal, manipulación genética y hormonal de alimentos, utilización de químicos para mayor rendimiento en cultivos en la producción agrícola y los alimentos transgénicos.

Finalmente, como la ética, la moral y el derecho siempre han sido disciplinas generadas por la actividad humana muy cercana e íntima en su correlación. La ética, dentro de su ideal, exige un esfuerzo continuo y en muchas ocasiones hasta excesivo; Mientras el derecho exige el mínimo de esfuerzo para su adhesión puesto define como el mínimo ético establecido para determinado grupo social. 
La ética impone sólo deberes de unilateralidad, mientras el derecho está marcado por la bilateralidad, imponiendo obligaciones jurídicas y concediendo derechos. La ética ciencia autónoma, resultante de la conciencia; El derecho ciencia heterónoma, se impone por la autoridad. La ética tiene un ámbito de actuación más restricto que el derecho, debido a la tendencia a disciplinar también cuestiones técnicas y económicas, pueden ser completamente extrañas o aislada a la moral. Finalmente, para la ética las sanciones impuestas son morales, para el derecho las sanciones son jurídicas (s, 2014 y Schaefer, 2017).

Por tal motivo, la bioética no debe ser considerada como una actividad o una disciplina aislada de estas dos últimas. Se puede entender la bioética en este caso como la respuesta a los avances biomédicos de las últimas décadas y de donde nace la necesidad de regular por parte del derecho, estas nuevas situaciones jurídicas engendrada por dichos avances; Entonces la sinergia entre la ética, la moral, la bioética y el derecho dan origen al bioderecho como una nueva forma jurídica para enfrentar y solucionar los conflictos de interés público relacionados con el derecho a la vida desde el nacimiento hasta la muerte, pero con un respeto a la dignidad de cada ser humano (Schaefer, 2017).

Es por esto que no pueden, ni deben ser tratadas como sinónimos la ética, la bioética y el bioderecho, a pesar de su intrínseca relación a la cual están ligados íntimamente, la ética sin el derecho pierde coercitividad y el derecho sin la ética pierde legitimidad. Tampoco se puede pretender desde los principios de la bioética, que las pretensiones de esta sean las misma que pregone el bioderecho. La bioética necesita de la reflexión ético-moral previa y del debate ciudadano, pero posteriormente requiere decisiones jurídicas, en aquellas aplicaciones biomédicas de las nuevas biotecnologías que no suelen plantear el problema de ser ilícitas sino más bien de cuáles son los límites a su licitud. 


\section{Biojurídica o bioderecho o biolegislación su conceptualización}

No cabe duda de que el prefijo "bio" está en boga, la percepción social de lo "bio" relacionado con las ciencias de la vida, la incidencia e incorporación de otras ramas científicas (sociología, derecho, economía, entre otros) donde destaca, como no podía ser de otro modo, la importancia actual de la investigación biomédica en las ciencias de la salud. Como puede observarse, también en el ámbito de las ciencias jurídicas como el derecho están surgiendo diversos términos que giran a la par de los nuevos conocimientos biotecnológicos y las nuevas prácticas y técnicas biomédicas, tanto para hacer alusión a aspectos o realidades precisas, como para exponer, más genéricamente, a la conexión o vínculos metodológicos de enlace de esta nueva disciplina científicas, como lo es la biojurídica, la biolegislación o el bioderecho (Mellano, 2008).

Ahora bien, junto al término bioderecho, no resulta infrecuente el empleo del término biojurídica. El primero se halla, por razones obvias, más cercano a la expresión anglosajona biolaw o bioethics law. El segundo procede de la doctrina italiana, suelen utilizar, indistintamente, ambos vocablos e, incluso, las expresiones biolegislación o biojurisprudencia (Flores, 2004).

En una primera visión, este hecho podría resultar intrascendente. Pero sin embargo da la impresión de que, parece posible advertir una mayor predilección por el término bioderecho o biolegislación, sobre todo en aquellos jurisconsultos que provienen de ramas específicas de la ciencia jurídica como: lo son el derecho constitucional, administrativo, mercantil, derechos humanos, penal, civil, entre otros., mientras la expresión biojurídica suele tener más connotación y coherencia con el ámbito filosófico del derecho.

Entonces se puede definir o conceptualizar a la biojurídica como el conjunto de normas integrales, nacen como una respuesta al nacimiento de la bioética, para proteger al ser humano de la aplicación de nuevas tecnologías, biomedicina, biotecnología o decisiones que tengan que ver con la vida. Por lo que el papel de la bioética debe ser por tanto la actuación ética en relación con la vida humana en el ámbito 


\section{Juan Carlos Araujo-Cuauro}

Telos Vol. 21, No. 3 (2019). 591-617

de la investigación y de la práctica médica.

Esta estudia la serie de comportamientos o conductas innatas e inmutables de las especies que forman un ordenamiento jurídico perfecto, bios y ius. Es un conjunto de normas de carácter inmutable con dos vertientes, que remite a la reflexión teórica por sí misma, sobre cuestiones bioéticas, adoptadas desde una perspectiva no ética, sino estrictamente jurídica, mientras la biolegislación es el resultado de la actividad del legislador llamado a traducir en normas legales los principios elaborados desde la biojurídica.

De este modo, la función de la biojurídica será, precisamente, la de justificar aquellos principios, no morales, sino propiamente jurídicos, en donde se apoyaría el bioderecho o la biolegislación.

La biojurídica es la respuesta desde el mundo del derecho al surgimiento de la bioética, como disciplina viene a confeccionar una respuesta social a las nuevas eventualidades de la bioética, no puede confinarse a legalizar a priori lo viable gracias a la invención científico tecnológica, en virtud del principio comúnmente compartido, y sobre en el cual descansa la ética como disciplina normativa, en donde no todo lo viable es por eso mismo lícito.

Esta viene tratando de argumentar y justificar los principios que secundan o apoyan el bioderecho o la biolegislación, permite acceder a una reflexión crítica y globalizada sobre lo implícito de las normas jurídicas. Por lo tanto, la bioética y la biojurídica están en una ajusta relación, la biojurídica viene a implantar límites a la aplicación de las ciencias biomédicas, anteponiendo el respeto a la vida y la tutela de la integridad física y moral de las personas.

Entonces la biojurídica es la rama de la bioética que se ocupa de la ordenación jurídica y la trascendencia de las aplicaciones jurídicas de la problemática bioética, constituyendo al mismo tiempo una reflexión crítica sobre las crecientes y fecundas relaciones entre la bioética y el derecho (Mazo, 2014). 
Es la ciencia cuyo objeto tiene la fundamentación y pertinencia de las normas jurídico positivas de "lege data" y de "lege ferenda" para lograr y verificar su adecuación a los principios y valores de la ética en relación con la vida humana

Es por esto atendiendo al contenido expuesto por los diversos autores, se podría afirmar, la biojurídica y el bioderecho se remitirían o emplazarían a niveles de conocimiento distintos.

En una visión general, el bioderecho abordaría el estudio de las realidades o fenómenos bioéticos, es decir, de los diversos conflictos surgidos en el ámbito de las relaciones humanas, como consecuencia de la incorporación de los nuevos aportes tecnológicos a las ciencias de la salud, desde la perspectiva de la ciencia jurídica (Parra, 2006).

Lo esencial, para ello, son aproximaciones desde el derecho positivo. Entonces estaríamos ante la presencia de una parte de la ciencia jurídica de la que, en realidad, siempre se han ocupado los juristas recordemos la Constitución bolivariana promulgada por la Asamblea Nacional Constituyente (1999), reconoce, en su artículo 84․, el derecho a la protección de la salud, desarrollado, entre otras leyes, en la Ley Orgánica de Salud.

Lo novedoso actual radicaría en el hecho, en las últimas décadas, la normativa legal referente a estas materias ha sufrido un notable desarrollo. Ello podría llevar a justificar la existencia de esta nueva disciplina, también denominada biolegislación.

Por su parte, la biojurídica abordaría, asimismo, cuestiones bioéticas, pero adoptándola desde una perspectiva distinta, la aportada por la filosofía jurídica o del derecho.

De este modo, la función de la biojurídica será, precisamente, la de justificar aquellos principios, no morales, sino propiamente jurídicos, en donde el bioderecho o la biolegislación se apoyarían.

En definitiva, la biojurídica llevaría a cabo una reflexión global y crítica sobre el bioderecho. En consecuencia, de esta disciplina se espera la no descripción fáctica de la legislación vigente en estas cuestiones. Dicha labor sería, en rigor, más propio del 
bioderecho o la biolegislación e incluso, de la biojurisprudencia (Aparisi, 2007).

A la biojurídica le corresponde al igual la filosofía del derecho, reflexionar sobre las normas vigentes, a la luz del principio de la dignidad humana y de los derechos humanos. Es este sentido, podríamos destacar otro reto de la biojurídica: incluir los derechos bioéticos en el contexto de los derechos humanos, garantizando al ser humano las condiciones para ejercer plenamente su identidad relacional y la propia humanidad (Maldonado, 2007).

En otras palabras, la bioética busca regular desde lo ético temas de alta complejidad como los ligados al inicio de la vida y la dignidad y el fin de la vida, es decir la muerte y la dignidad póstuma, esta busca la reducción de los conflictos generados por los dilemas resultantes de los avances de biotecnología en el campo biomédico de tal modo se privilegie la coexistencia de la humanidad.

El derecho procura a través del bioderecho, el cual tiene una base normativa e impositiva ejercer una regulación jurídica, la cual sea capaz de dar protección legal o soluciones normativas a los individuos de las diversas cuestiones jurídicas, derivadas de los grandes dilemas resultantes de la biotecnología relacionados con la medicina (Schaefer, 2017).

La imperiosa necesidad de aproximar los impresionantes progresos resultantes de las biotecnociencias básicas aunado a los conocimientos médicos, son imprescindible para el tratamiento adecuado de las cuestiones emergentes en especial, las provocadas por los grandes cambios en las relaciones humanas, los avances biomédicos generan situaciones las cuales serían indiscutibles, desde el punto de vista ético, para discutir con mayor criticidad las nuevas investigaciones, en un contexto bioético-biojurídico marcado por el desarrollo de las nociones de autonomía y libertad de conciencia.

La bioética como disciplina señala situaciones de cuestiones emergentes, donde sugieren soluciones éticas. Mientras al derecho como ciencia social le compete 


\section{La biojurídica o el bioderecho como mediador de los nuevos dilemas biomédicos}

dar soluciones jurídicas a los conflictos bioéticos, estableciendo la protección de los individuos en su integralidad, a través de un sistema de principios y valores considerados universales y vinculantes.

Desde su inicio como disciplina de la moral, la bioética propone reflexiones éticas; el derecho como ciencia propone reflexiones jurídicas a partir de su mayor premisa o valor que tiene una persona como la protección a su dignidad.

En cuanto al derecho médico desde su génesis tradicional, hacía referencia o estaba relacionado con la actividad profesional del personal sanitario (médico y otros profesionales de la salud) con el sistema de salud y su organización médico asistencial, con los pacientes y usuarios de la red de salud, pública o privada, y con otros profesionales que ejercen sus actividades en el campo de la salud y, sobre todo, de las responsabilidades jurídico legales generadas debido a las acciones imprudentes o negligentes, como consecución de la mala praxis profesional.

Entonces el derecho médico o derecho biomédico, derecho biotecnológico (health law, health care law), es utilizado en algunos sistemas jurídicos en sus ordenamientos como sinónimo de bioderecho (biolaw). El derecho médico tradicional se dedica a los aspectos jurídicos vinculados al ejercicio de la medicina y demás profesiones ligadas directamente a la salud. Pero no obstante el bioderecho en la actualidad comprende, las implicaciones jurídicas de los avances de las denominadas ciencias biotecnológicas y biomédicas en su interactuar con el ser humano (Schaefer, 2017 y Beltrán 2019).

En la legislación venezolana en su ordenamiento jurídico no contempla el derecho médico, ni mucho menos el bioderecho, en los aspectos jurídicos vinculados al ejercicio profesional de la medicina o a las implicaciones jurídicas de los avances biomédicos tecnológicos.

Aunado a lo antes expuesto el bioderecho en su surgimiento como nueva disciplina de las ciencias jurídicas, desde un principio debe tener mucha claridad en cuáles son las funciones de este derecho como ciencia social humana.

Esto radica en el hecho de poder explicar cualquier teoría del derecho, es 
necesario e indispensable conocer sus funciones, si bien como en el bioderecho, no está de más tener en cuenta cuáles son sus bases para su desarrollo, son las mismas que dieron origen a otras ramas del derecho y por esa similitud en algunas circunstancias tiene relación con otras disciplinas. (Becerra, 2013).

Algo característico del conflicto surgido en área de las ciencias médicas como la relación médico-paciente, dentro de este convergen muchas normas, las cuales hay que tener y tomar en cuenta al momento de su análisis, confluyen leyes de la naturaleza representada desde un inicio por la vida y asimismo normas prescriptivas al encontrarse la vida protegida por el Estado. Al analizar ambas y buscar su origen y razón de ser, entran en juego las leyes de la lógica (Luelmo, 2007).

El bioderecho constituye una nueva forma de afrontar la búsqueda de solución a los conflictos médico legales planteados en esta era posmoderna producto de los avances biotecnológicos, como axionar de la responsabilidad profesional médica, como especialidad jurídica de relevada importancia para el derecho de daños y para la reflexión de las implicaciones legales de las nuevas tecnologías sobre la vida. La protección del derecho a la vida se juega también, y decisivamente, en la praxis jurídica, en la deducción y en la evolución del proceso de recreación del derecho que procede a la resolución de casos en concreto (Beltrán 2019).

El término biolegislación parece hacer referencia a la dimensión exclusivamente normativa o positiva de las investigaciones biotecnológicas y biomédicas y de sus aplicaciones médico asistencial o comercial.

La bioética como ciencia tiene una historia corta y más aún en nuestro país Venezuela, esa historia apenas se inicia o en el peor de los casos aún no se ha iniciado; situación ubicada directamente en el escenario del bioderecho, es una consecuencia del origen de la bioética, es decir el surgimiento del bioderecho es más reciente al de la bioética.

El bioderecho es una especie de respuesta tardía por parte de los juristas y 


\section{La biojurídica o el bioderecho como mediador de los nuevos dilemas biomédicos}

legisladores, se debe en parte a contradicciones entre las ciencias, un ejemplo de ello es la contradicción perpetua entre la Medicina y el Derecho.

El bioderecho es el conjunto de normas jurídicas de interés público, íntimamente ligadas a la protección de la vida desde su inicio hasta su final respetando la dignidad humana.

Este viene de alguna manera a solucionar los conflictos desde el planteamiento ético, con el soporte o aval de la ciencia y bajo el marco de un derecho cercano a la sociedad cuyo referente último radica en el imperativo sustentado por los Derechos Humanos.

Con el avance tecnológico de la ciencia, en este recién nuevo siglo que comienza (Siglo XXI) sobre todo lo biotecnológico de proporciones impensable hace pocas décadas atrás. Es por ello el bioderecho ha querido erigirse como la esfera del derecho regulador de las nuevas tecnologías biomédicas surgidas en la sociedad posmoderna (Flores, 2004).

Su interés es eminentemente normativo y, por consiguiente, como se había expresado ante, negativo, se concentra en el estudio de los riesgos, peligros y amenazas de las nuevas biotecnologías sobre el campo de la medicina, aplicadas a los seres y sistemas vivos implican o acarrean situaciones problemáticas con consecuencia médico legales.

Bajo este concepto podemos encontrar todo aquello que ponga en riesgo la vida, la libertad, la dignidad y los demás principios definido como los rectores de la sociedad moderna los cuales se vuelven objeto de regulación normativa y jurídica.

Es necesario recordar y tener siempre bien definido uno de los propósitos del derecho, es señalar las vías jurídicas procesales adecuadas para las relaciones interpersonales entre las personas, transcurran en un ambiente de orden para evitar una colisión entre intereses o derechos y obligaciones determinadas (Parra, 2006).

En este nuevo rol del derecho, de abordar los temas bioéticos y los nuevos conceptos jurídicos, como el de la persona, están en plena reelaboración, con el ánimo de trazar los lineamientos para que exista compatibilidad entre los avances 
biotecnológicos y de los valores asegurar como lo son el respeto y el reconocimiento por los seres humanos.

El bioderecho sistematiza y da coherencia jurídico-ética a las nuevas realidades emergentes modernas sobre la vida y la sociedad como figuras jurídicas poco observadas y con abismos legales en el marco jurídico venezolano. El Bioderecho es un área de la justicia compuesta por los diversos casos y soluciones producidas particularmente en el desarrollo de la vida humana (Moran, 2006).

Para entender y por si comprender el campo de acción del bioderecho, es necesario tener constantemente presente las vicisitudes dadas entre la vida humana, los fenómenos de crisis y de "revolución", quizás los más grandes de todo el desarrollo humano, generados por el sorprendente impacto técno-cientifico actual.

Las ideas y reflexiones expresadas conllevan a plantear cuales son los retos a enfrentar por el bioderecho en la sociedad actual. Lo primero a enfrentar por el bioderecho, es la necesidad para acometer un diálogo interdisciplinario con otras áreas del saber. El respectivo diálogo no debe tener como propósito el no torcer un conocimiento en beneficio de otro; su fin debe ser el de enriquecer la visión de los múltiples fenómenos y problemas sobre los cuales nos movemos en el día a día (Parra, 2006).

El bioderecho es una nueva rama científica interdisciplinaria, dotada con objetivos, principios y con finalidades propias dedicada a bordar de manera inédita y de forma novedosa el intrincado y apasionante campo relacionado con la vida, con la reproducción humana y la muerte determinando sus alcances y sobre todo sus límites.

El bioderecho, en muchas situaciones, surge como respuesta a los avances del progreso científico y tecnológico vinculados con la problemática de los seres vivientes, y representa una simbiosis entre la vida y el comportamiento humano en su entorno natural, que se relaciona con todo aquello relativo a la vida y la dignidad (Martínez, 2013). 


\section{La biojurídica o el bioderecho como mediador de los nuevos dilemas biomédicos}

Se puede entender al bioderecho como una respuesta jurídica a los avances científicos y tecnológicos de los últimos años y la necesidad de regulación de las situaciones nuevas de dichos avances representan; los reclamos del respeto a la dignidad y la libertad humana encuentran en el bioderecho un excelente aliado (Mazo, 2014).

Los avances de la ciencia, la posibilidad de intervenir en la entidad constitutiva del ser humano, la delgada línea que separa ciencia y tecnología de la intimidad, el conflicto abierto entre lo natural y lo artificial y el debido respeto a la dignidad humana, nos plantea un abrumador e interrogante panorama, se constituye en la razón de ser y en el punto de partida de la ciencia del bioderecho.

Es decir, en todo aquello precisamente en donde exista la intervención humana, genera la alteración de los procesos naturales de generación y conformación de la vida humana, animal y vegetal, hasta hace pocas décadas un territorio vedado para las ciencias como la biología, la ética, la filosofía, y la jurídica.

La trascendencia de los nuevos retos del milenio, en definitiva, han propiciado la eclosión del bioderecho como disciplina y el crecimiento, en las dos últimas décadas, ha experimentado la relación entre la biología, la tecnología, la medicina, la ética y el derecho.

La sociedad, el ser humano y el bioderecho, se preguntan si frente al creciente poder de intervención de la ciencia y de la tecnología en la vida humana, todo lo materialmente posible es éticamente conveniente y, si lo es, dentro de qué límites jurídicos licito es aceptable (Aparisi, 2007).

En la actualidad, la interconexión e interdependencia entre las ciencias es cada vez más evidente y las soluciones justas y respetuosas con la identidad (humana, natural y cultural) de nuestro entorno exigen del concurso de un nuevo estilo de trabajo.

Ante una controversia que afecta a una temática determinada; Ante un problema relacionado con las nuevas tecnologías, la privacidad o la intimidad; o ante un problema de dignidad, no sólo interviene la ciencia y sus conocimientos avanzados; también interviene la ética, interrogada por los límites y las realidades transcendentes comprometidas en la decisión a tomar; y también lo hace el derecho estableciendo los 


\section{Juan Carlos Araujo-Cuauro}

Telos Vol. 21, No. 3 (2019). 591-617

cauces de convivencia necesarios, las garantías de los derechos reconocidos, los deberes asignados y el mínimo común denominador mediante el cual el derecho, la ética y la ciencia tienden a convergen en la búsqueda de soluciones más conscientes y justa para la sociedad (Maldonado, 2007).

Para poder ser una ciencia el bioderecho, y tener un respaldo exitoso, este haz de respetar ciertas reglas básicas como lo son:

1. Debe fundamentarse en una ética capaz de admitir una gran variedad de corrientes del pensamiento universal, proyectándose desde una ética aplicada de carácter civil sustentada en la racionalidad humana.

2. Debe atender al estado actual de la ciencia, siendo capaz de valorar el alcance de los resultados que ésta aporta.

3. Debe realizar los análisis jurídicos con carácter interdisciplinar.

4. Debe, en fin, sustentarse en los Derechos Humanos. Este es el origen y fundamento último a referirse en todo momento.

En ellos se condensan los ámbitos de reconocimiento y protección, permiten fortalecer la dignidad del ser humano. El bioderecho ha de contribuir a la aplicación efectiva de estos derechos y a la profundización en el desarrollo de su contenido aplicado a las ciencias de la vida. En definitiva, el bioderecho es una ciencia, ha de tener, como eje argumental de todas sus reflexiones, la sensibilidad hacia el ser humano (Aparisi, 2007).

Es una ciencia, desde la objetividad de sus planteamientos y resultados, no puede dejar de mirar a quienes son sus destinatarios; y en ellos, lo que trasluce es dignidad, realización personal, derecho a vivir y a morir con dignidad y a ser respetados socialmente en su autonomía y en su principio de libertad de conciencia, en cuanto este respeto se formula con reciprocidad.

Se debe hacer énfasis en el hecho de la función del bioderecho en el ordenamiento jurídico de cualquier país, y en este caso en la legislación venezolana, no 


\section{La biojurídica o el bioderecho como mediador de los nuevos dilemas biomédicos}

debe tender a normar todos los aspectos de la bioética sino a brindar el marco dentro del cual se puedan resolver y/o solucionar conflictos entre la ciencia médicas y la ciencia jurídicas y los avances biotecnológicos, el no contar con este marco jurídico puede sobrevenir en el individuo una indefensión debido a la falta medios legales para los grandes vacíos legales, que puedan actuar en perjuicio de sus derechos como persona independientemente de la intencionalidad.

Por el contrario, si el bioderecho se encarga de crear los mecanismos e instituciones para la solución de los problemas bioéticos siempre tendrán las bases para tratarlos y permitirá una constante actualización, entonces no se trata de la creación de una norma nueva o no tratada dentro del ordenamiento jurídico, sino a partir ordenamiento positivo crear las normas que permitan regular esas nuevas situaciones dentro de la sociedad como son las cuestiones bioéticas y biojurídicas (Díaz et al, 2007).

Finalmente, ya nadie puede ignorar que la bioética como disciplina analizada desde los principios bioéticos de justicia, equidad, autonomía, dignidad, no maleficencia, beneficencia son sin duda idearios que conforman y modelan el pensamiento jurídico y bioético. Fundamentos que son compartidos en su mayoría por el derecho. Las declaraciones bioéticas son las manifestaciones más importantes de esta nueva dinámica jurídica se ha dado en denominar: bioderecho, biojurídica, biolegislación.

\section{Conclusiones}

Si bien ética la bioética y el derecho son disciplinas diferentes, se encuentran internamente relacionadas, en el planteamiento que hacen con referencia a la correlativa influencia de la ética, a la bioética, y del derecho al bioderecho.

La ética, la bioética y el derecho no pueden o antes las nuevas relaciones sociales permanecer inerte ante los resultantes avances de la biotecnología biomédica, es un error común el querer solucionar problemas bioéticos como problemas jurídicos.

Es indiscutible la íntima relación entre la bioética y el derecho, ambas ciencias se han unido para dar pie a una nueva disciplina jurídica denominado bioderecho que 
viene a sistematizar y dar coherencia a lo ético-jurídico de las nuevas realidades emergentes ante el desarrollo científico tecnológico que ha alcanzado la humanidad, por lo que se encarga de establecer límites y regulaciones en el ámbito de las ciencias de la vida y de la salud.

Entonces la bioética y el bioderecho han venido recorriendo un largo y estrecho camino en común, por lo que a lo largo de este recorrido ambos se han nutrido de forma recíproca. Así como la bioética es la respuesta desde lo ético moral, la biojurídica o bioderecho o biolegislación es la respuesta desde el mundo del derecho al surgimiento de la bioética.

Los dilemas bioéticos del bioderecho no se solventan inicialmente por la vía de la moralidad, sino que es necesaria previamente una amplia discusión ética para luego crear leyes tan complejas como las relacionadas con la ética del inicio y final de la vida. Así, pues, hay que distinguir entre bioética como deliberación y el bioderecho como legislación.

\section{Referencias bibliográficas}

Aguiar-Guevara, Rafael. (2008). Tratado de Derecho Médico (segunda edición). Legislec Editores. Venezuela.

Aparisi Miralles, Ángela. (2007). Bioética, bioderecho y biojurídica (reflexiones desde la filosofía del derecho). Anuario de filosofía del derecho, 24, 63-84. Extraído de: https://dialnet.unirioja.es/servlet/articulo? codigo=2769929. Consulta: $\underline{20 / 06 / 2018}$.

Asamblea Nacional Constituyente (1999). Constitución Nacional de la República Bolivariana de Venezuela. Gaceta Oficial 36.860. Venezuela.

Becerra-Ramírez, Manuel. (2013). Derecho y Medicina. ASPE. México.

Beltrán Aguirre, Juan Luis. (2014). Bioética y derecho biomédico: principios informantes. su reflejo en la normativa y en la práctica asistencial. Enfrentamientos, prevalencias y transgresiones. Derecho y salud, 24(1): 21-58. Extraído de: https://www.ajs.es/descarga/attach/606. _Consulta: 07/05/ 2019.

Casado González, María. (2002). ¿Por qué bioética y derecho? Acta bioethica, 8(2): Extraído de: 183-193. https://dx.doi.org/10.4067/S1726-569X2002000200003. 
Consulta: 07/07/ 2018.

Celi Frugoni, Alina. (2015). Conflictos bioéticos y biojurídicos en la salud pública Global. Revista Direitos Humanos E Democracia, 3(5), 29-46. Extraído de: https://doi.org/10.21527/2317-5389.2015.5.29-46. . Consulta:07/07/2018.

Cornejo Plaza, María Isabel. (2015). Naturaleza jurídica de las Declaraciones Internacionales sobre Bioética. Revista de Bioética y Derecho, (34), 26-36. Extraído de: https://dx.doi.org/10.1344/rbd2015.34.12064. Consulta: 19/05/19. Díaz-Ambrona Bardají, María Dolores. Serrano Gil, Alfonso, Fuentes Rocañin José

Carlos. Hernández Díaz-Ambrona, Pablo (2007). Introducción a la Medicina

Legal. Ediciones Díaz Santos. España.

Flores Trejo, Fernando. (2004). Bioderecho. Porrúa. México.

Gutiérrez Samperio, Carlos y Vila-Coro Barrachina, María Dolores. (2007). Bioética y biojurídica. Revista internacional de bioética, deontología y ética médica, 18(1):53-70. Extraído de https://dialnet.unirioja.es/servlet/articulo?codigo=2770507._Consulta:_07/07/ 2018.

Gracia, Diego. (1989). Fundamentos de Bioética. (tercera edición). Editorial Triacastela. Madrid.

Hooft, Pedro Federico. (2002). BIOÉTICA Y JURISPRUDENCIA. Acta bioethica, 8(2), 211-237. Extraído de https: //dx.doi.org/10.4067/S1726-569X2002000200005.

Luelmo Domínguez, Andrés. (2007). Derecho Sanitario y Responsabilidad Médica. Editorial Lex Nova S.A. España.

Maldonado Castañeda, Carlos Eduardo. (2007 ene). Bioética, biopolítica y bioeconomía: panorama práctico sobre la bioética. Bioética y biojurídica, 4352. Extraído

http://www.scielo.org.co/scielo.php?script=sci_nlinks\&ref=000181\&pid=S190 9-0455201400020000700013\&lng=en. Consulta: 10/06/ 2018.

Martínez Bullé-Goyri, Víctor. (2013). Reflexiones sobre la dignidad humana en la actualidad. Boletín mexicano de derecho comparado, 46(136), 39-67. Extraído de: https://doi.org/10.1016/S0041-8633(13)71121-9. Consulta: 07/07/ 2018.

Mazo Álvarez, Héctor Mauricio. (2014). El bioderecho: La respuesta jurídica a los problemas que plantea la bioética. Producción + Limpia, 9(2), 74-88. Extraído de: $\quad$ http://www.scielo.org.co/scielo.php?script=sci_arttext\&pid=S190904552014000200007\&lng=en\&tlng=. Consulta: 07/07/ 2018.

Mellano Ruiz, Lorenzo. (2008). LA BIOSEGURIDAD COMO CONCEPTO JURÍDICO. Revista catalana de dret públic, 36: 19-54 Extraído de http: //www.revistes.eapc.gencat.cat/index.php/rcdp/article/download/2149/n36mellado-es.pdf. Consulta: 18/05/ 2019.

Moran González Luis. (2006). De la Bioética al Bioderecho. Ediciones Dykinson. España.

Parra Tapia, Ivonne. (2006). Consideraciones biojurídica sobre la vida en el embrión humano. Dikaiosyne,16:35-53.Extraído 
Juan Carlos Araujo-Cuauro

Telos Vol. 21, No. 3 (2019). 591-617

http://www.saber.ula.ve/bitstream/handle/123456789/19096/articulo3.pdf?seq uence=2\&isAllowed=y. Consulta: 10/08/ 2018.

Schaefer Rivabem, Fernanda. (2017). Bioderecho: ¿una disciplina autónoma? Revista Bioética, 25 (2), 282-289. Extraído de https://dx.doi.org/10.1590/198380422017252188. Consulta: 10/06/ 2018.

Vila Coro, María Dolores. (2005). El marco jurídico de la bioética. Cuadernos de Bioética, 16(3), 313-321. Extraído de: http://www.redalyc.org/articulo.oa?id=87512622001. Consulta: 10/06/2018. 